\title{
Effect of correlations on signal transmission in a population of spiking neurons
}

\author{
Mona Spiridon $^{\mathrm{a}, *}$, Carson C. Chow ${ }^{\mathrm{b}}$, Wulfram Gerstner ${ }^{\mathrm{a}}$ \\ ${ }^{a}$ Center for Neuromimetic Systems, Swiss Federal Institute of Technology, Lausanne, EPFL-DI, \\ MANTRA, LAMI, CH-1015 Lausanne EPFL, Switzerland \\ ${ }^{\mathfrak{b}}$ Department of Mathematics, University of Pittsburgh, PA 15260, USA
}

Accepted 13 January 2000

\begin{abstract}
Biological neurons are noisy. Nevertheless, they are able to transfer information in a very reliable manner. We address the problem of signal transmission reliability using a network of stochastic neurons of the integrate-and-fire type. We have derived an analytical expression of the noise power and the signal-to-noise ratio as a function of different network parameters. If firing times of different neurons are anticorrelated, signal transmission can be improved over a broad frequency range beyond the single-neuron firing rate. (C) 2000 Elsevier Science B.V. All rights reserved.
\end{abstract}

Keywords: Population dynamics; Spiking neurons; Noise shaping; Information transmission

\section{Introduction}

Cortical neurons exhibit a high degree of variability in the discharge patterns [7]. On the other hand, external signals are transmitted with high fidelity and high speed [9]. Here we investigate how a population of noisy spiking neurons can transmit a rapidly changing signal in a reliable manner. Having a populations of neurons with similar properties is a common organizational principle in many areas of the brain. If $N$ neurons transmit the same signal, averaging over $N$ independent neurons improves the signal-to-noise ratio by a factor proportional to $1 / \sqrt{N}$.

\footnotetext{
* Corresponding author. Tel.: + 41-21-6933-910; fax: + 41-21-6935-263.

E-mail address: mona.spiridon@epfl.ch (M. Spiridon).
} 
In biological system, neurons are connected to each other. We study how connections might affect signal transmission. To do so, we assume that information is transmitted through the activity of the neuronal population defined as

$$
A(t)=\lim _{\Delta t \rightarrow 0} \frac{1}{\Delta t} \frac{n_{\text {act }}(t ; t+\Delta t)}{N},
$$

where $N$ is the population size and $n_{\text {act }}(t ; t+\Delta t)$ the number of neurons that fire during the interval $\Delta t$. The activity corresponds to a spatially averaged rate. In contrast to a temporally averaged firing rate code, the population rate code can respond rapidly to a change in the input $[2,4,10,11]$.

It has been shown that, for constant stimuli, certain types of correlation can increase the amount of information transmitted by a population of neurons coding a feature variable $[1,12]$. Here, we use a homogeneous pool of neurons to look at the non-zero frequency correlations. We show that anticorrelations, produced by inhibitory connections, can lower the noise level in a certain frequency band and hence improve signal transmission $[5,8]$.

\section{The model}

We consider a homogeneous, fully connected network. Neurons are described by the spike response model [3], which is a variant of the integrate-and-fire model. Spikes from presynaptic neurons induce, after an axonal delay $\Delta_{\mathrm{ax}}$, a membrane depolarization represented by the kernel $\varepsilon\left(t-t_{j}^{(\mathrm{f})}-\Delta_{\mathrm{ax}}\right)$, where $t_{j}^{(\mathrm{f})}$ is the presynaptic firing time of neuron $j$. The absolute and relative refractory period is described by the kernel $\eta\left(t-t_{i}^{(\mathrm{f})}\right)$ which depends on the difference between the present time $t$ and the last firing $t_{i}^{(\mathrm{f})}$ of the neuron under consideration. The total potential $u_{i}(t)$ of neuron $i$ can be written as [3]

$$
u_{i}(t)=\eta\left(t-t_{i}^{(\mathrm{f})}\right)+h(t)
$$

with the postsynaptic potential $h(t)$

$$
h(t)=\frac{J}{N} \sum_{t_{j}^{(\mathrm{f}}} \varepsilon\left(t-t_{j}^{(\mathrm{f})}-\Delta_{\mathrm{ax}}\right)+h_{\mathrm{ext}}(t),
$$

where the sum runs over all firing times and all presynaptic neurons and is weighted with a coupling factor $J / N$. In addition, all neurons receive the same external input $h_{\text {ext }}(t)$. The kernels $\eta$ and $\varepsilon$ can be chosen arbitrarily. In our simulations we take an exponential function for $\eta$ and an $\alpha$-function for $\varepsilon$. In this case, the spike response model is a good approximation to the integrate-and-fire model.

A fully connected integrate-and-fire-type network will tend to fire in a synchronous manner. In order to avoid synchronous firings in the network, noise is introduced as follows: at each time step the neuron has a spiking probability density $\rho(t)$ that depends on the distance between the momentary value of the membrane potential and the threshold (see the appendix). It has been shown that this intrinsic noise model, that 
we call noisy threshold model, can reproduce quite well the variability in the spike trains of cortical neurons $[2,6]$. We say that the neurons in our network fire asynchronously if, for constant input, the population activity fluctuates around a constant mean. The advantage of asynchronous firings is that it allows a rapid response to a change in the stimulus $[2,11]$. With a sufficient amount of noise, the system is guaranteed to be in a state of asynchronous firing.

\section{Results}

To study the signal transmission properties of a population of spiking neurons, we have developed a theory for a fully connected homogeneous network of stochastic neurons [2]. If the population is infinite and in the absence of any external stimuli, the activity is constant over time (asynchronous firing). Due to the finite numbers of neurons, the population activity is fluctuating. We have found an analytical solution of the spectrum of the noise due to finite size effects [8].

The reliability of signal transmission is characterised by the signal-to-noise ratio $A_{\text {signal }}(\omega) / A_{\text {noise }}(\omega)$, where $\omega$ is the frequency, $A_{\text {signal }}(\omega)$ is the Fourier transform of the output signal and $A_{\text {noise }}(\omega)$ is the amplitude of the noise at frequency $\omega$. A sketch of the derivation of the noise spectrum and signal to noise ratio can be found in the appendix.

The theory is in good agreement with the simulations and remains valid for partially connected networks or for inhomogeneous neuronal networks where each neuron has a different spiking probability (Fig. 1). The anticorrelations in the firings shift the noise power from low to high frequencies (Fig. 1b). Thus, the anticorrelations, which arise from the inhibitory connections, allow a more reliable transmission of low-frequency signals. For a low-level activity, this reduction can go beyond the single neuron firing rate (see [8]).

The effect of inhibition on signal transmission can be seen as an increase in the dynamic range of the transmitted signal. Fig. 2 shows two populations where each neuron receives an external input $h_{\text {ext }}(t)$ that is modulated at $20 \mathrm{~Hz}$. In the first population, the coupling is excitatory $(J=2)$ whereas in the second one, the coupling is inhibitory $(J=-5)$. The amplitude of the input signal is the same in the two populations. With excitatory connections, the output signal is clearly in a nonlinear regime, whereas with anticorrelations, the activity shows nice oscillations at the input frequency. Thus, inhibitory connections allow a wider range of signal amplitude to be transmitted without important distortions.

\section{Conclusion}

We have derived an analytical expression of the noise spectrum and the signal-tonoise ratio for a population of integrate-and-fire neurons in a fully connected and homogeneous network. This simple network model captures the main features of the noise in the neuronal population. Redundancy in the network improves signal 

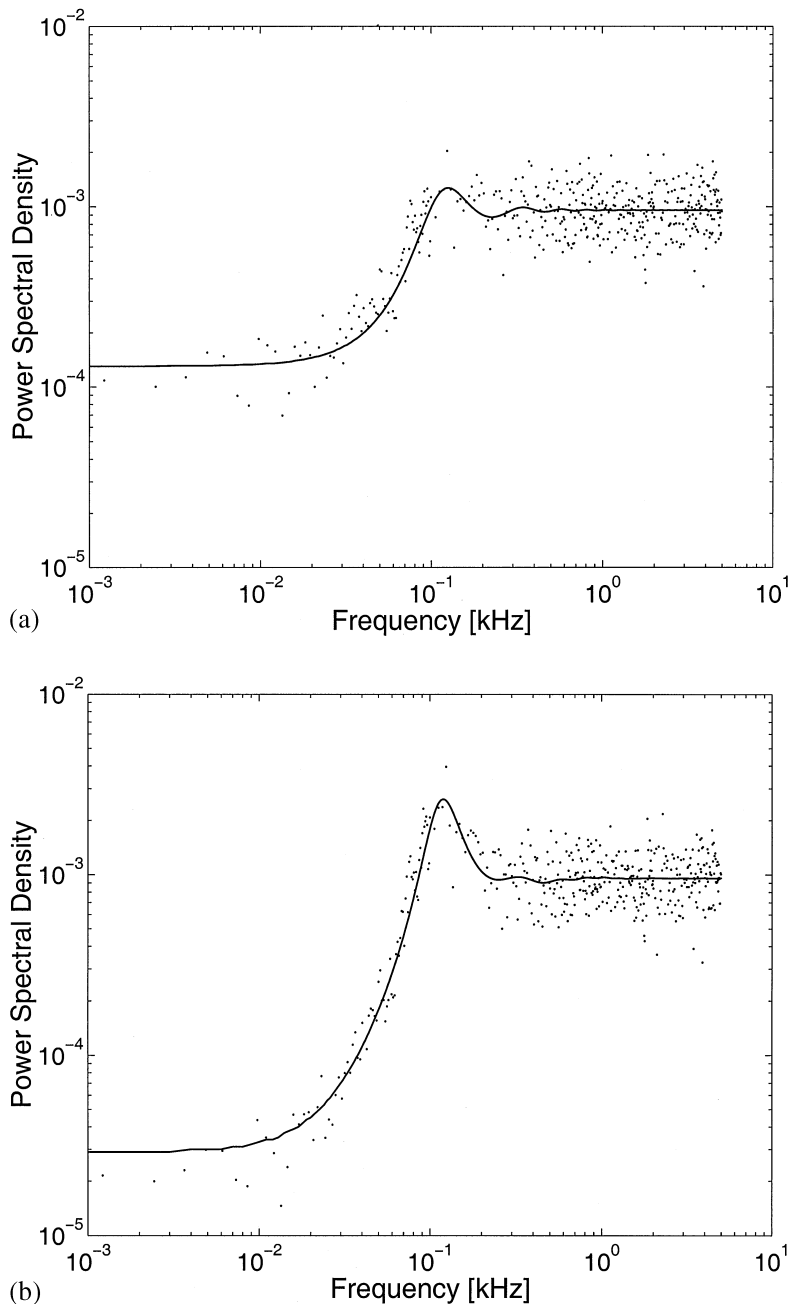

Fig. 1. Theoretical noise spectrum of a homogeneous network (solid line) and noise spectrum obtained from simulations (points) of an inhomogeneous network with 1000 neurons firing with a rate of $100 \pm 25 \mathrm{~Hz}$. (a) No coupling $(J=0)$ and (b) inhibitory coupling $(J=-5)$. The axonal delay is equal to $1 \mathrm{~ms}$ and the refractory function $\eta$ is exponential with a time constant of $4 \mathrm{~ms}$. The membrane and synaptic time constants are equal to $4 \mathrm{~ms}$. In order to keep the mean activity of the network to $100 \mathrm{~Hz}$ in the two cases, we adjust the constant term of the external input. In both theory and simulations, we use a spiking probability density $\rho$ that depends linearly on the input.

transmission. We found that further improvement is made possible by anticorrelations in the firings. Indeed, due to inhibition, noise can be reduced in the lowfrequency band. As a consequence, the network can increase its dynamic range for signal transmission. In addition to stabilising the network activity, inhibition might be useful to transmit signals more reliably. 


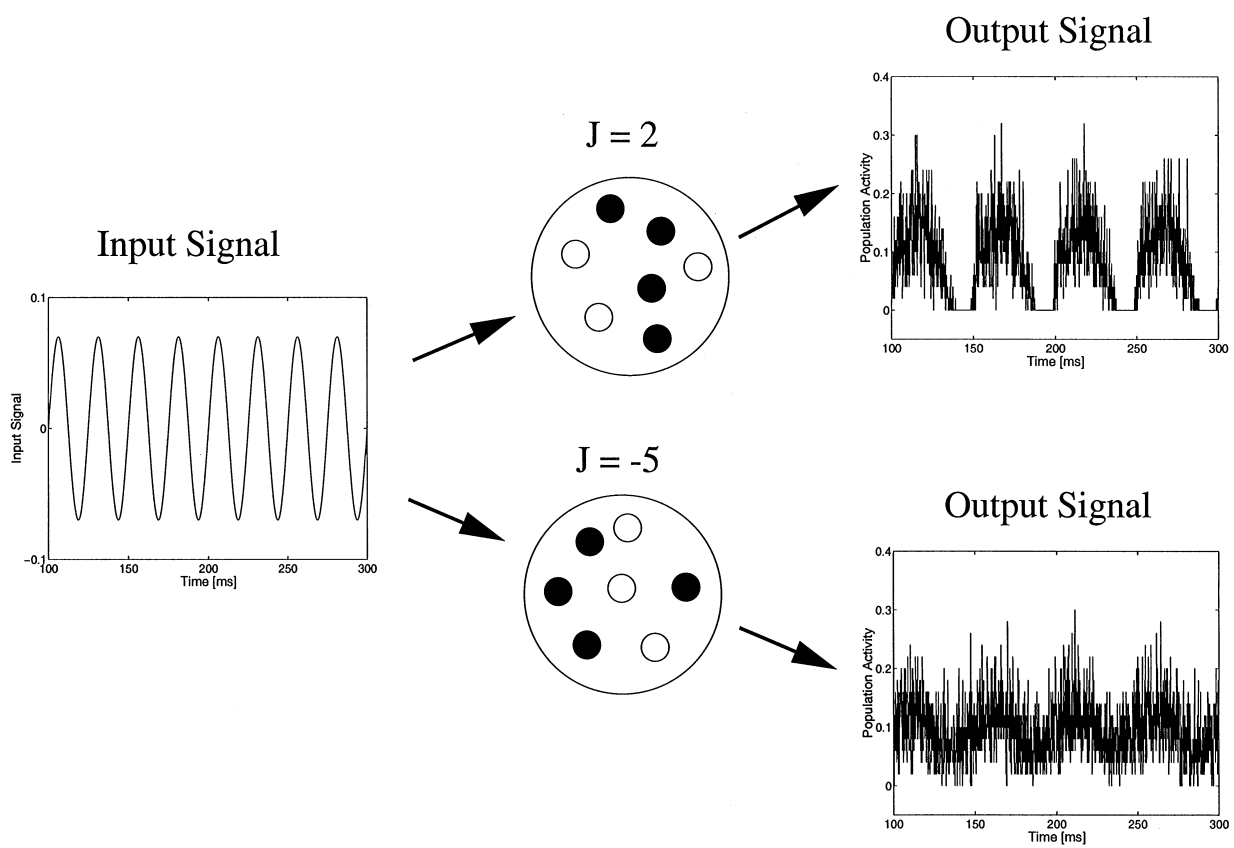

Fig. 2. An input current is given to two populations of neurons. One has only excitatory connections $(J=2)$ and the other is fully inhibitory $(J=-5)$. In the first population the output activity shows clearly distortion of the input signal whereas the inhibitory population is still able to reproduce correctly the input signal. The parameters of the network are the same as those of Fig. 1.

\section{Appendix}

The population activity dynamics in a homogeneous spiking neuron network can be written as [2]

$$
A(t)=\int_{-\infty}^{t} P_{h}(t \mid \hat{t}) A(\hat{t}) \mathrm{d} \hat{t}
$$

where the kernel $P_{h}(t \mid \hat{t})$ is the probability density that a neuron that has spiked at time $\hat{t}$ and that has a postsynaptic potential $h(t)$ will produce another spike at time $t$. The present activity $A(t)$ depends on the past activity $A(\hat{t})$ for $\hat{t}<t$. The probability density $P_{h}(t \mid \hat{t})$ can be expressed as a function of the escape rate $\rho(t)[2]$

$$
P_{h}(t \mid \hat{t})=\rho(t) \exp \left[-\int_{\hat{t}}^{t} \rho(s) \mathrm{d} s\right],
$$

where $\rho(t)=f[u(t)-\vartheta]$ is some function of the momentary distance between the membrane potential $u(t)$ and the threshold $\vartheta$. For $N \rightarrow \infty$ and sufficiently high noise, 
the activity $A(t)$ tends to a fixed point $A_{0}$ (see [2]). Due to the finite number of neurons, $A(t)$ will fluctuate around $A_{0}$. Linearization of Eq. (A.1) gives an expression of the signal spectrum (in the limit of $N \rightarrow \infty$ ) [2] and the noise power [8]

$$
\begin{aligned}
\left|\tilde{A}_{\text {signal }}(\omega)\right|^{2} & =\frac{A_{0}^{2}|\tilde{\mathscr{L}}(\omega)|^{2}}{\left|\tilde{S}_{h_{0}}(\omega)-J A_{0} \tilde{\mathscr{L}}(\omega) \tilde{\varepsilon}(\omega)\right|^{2}}\left|\tilde{h}_{\text {ext }}(\omega)\right|^{2}, \\
\left|\tilde{A}_{\text {noise }}(\omega)\right|^{2} & =\frac{\sigma^{2} A_{0}^{2}|\tilde{\mathscr{F}}(\omega)|}{\left|\tilde{S}_{h_{0}}(\omega)-J A_{0} \tilde{\mathscr{L}}(\omega) \tilde{\varepsilon}(\omega)\right|^{2}}|\tilde{\xi}(\omega)|^{2} .
\end{aligned}
$$

The signal-to-noise ratio is thus given by the ratio between the output signal (Eq. (A.3)) and the noise term (Eq. (A.4)):

$$
|\operatorname{SNR}(\omega)|^{2}=\frac{|\tilde{\mathscr{L}}(\omega)|^{2}}{\sigma^{2}|\tilde{\mathscr{F}}(\omega)|^{2}|\tilde{\xi}(\omega)|^{2}}\left|\tilde{h}_{\mathrm{ext}}(\omega)\right|^{2},
$$

where $\sigma^{2}=\left(1 / A_{0} N\right)$ and $N$ is the number of neurons in the population. For finite time step $\Delta t,|\xi|^{2}=1 / \Delta t . J$ is the coupling strength and $h_{0}$ is the constant term of the postsynaptic potential $h(t)=h_{0}+\Delta h(t)$. The tilde denotes the Fourier transform and the functions $S(t), \mathscr{F}(t)$ and $\mathscr{L}(t)$ are defined as

$$
\begin{aligned}
& S_{h_{0}}\left(t-t^{\prime}\right)=1-\int_{t^{\prime}}^{t} P_{h_{0}}\left(s \mid t^{\prime}\right) \mathrm{d} s, \\
& \mathscr{F}(t)=\mathscr{H}(t) \int_{t}^{\infty} \rho_{h_{0}}(s-t) S_{h_{0}}(s) \mathrm{d} s, \\
& \mathscr{L}(t)=\mathscr{H}(t) \int_{t}^{\infty}\left[\frac{\mathrm{d}}{\mathrm{d} h_{0}} \rho_{h_{0}}(s-t)\right] S_{h_{0}}(s) \mathrm{d} s,
\end{aligned}
$$

where $\mathscr{H}(t)$ is the Heaviside function, $\mathscr{H}(t)=1$ for $t>0$ and 0 elsewhere. The details of the development can be found in [8].

\section{References}

[1] L.F. Abbott, P. Dayan, The effect of correlated variability on the accuracy of a population code, Neural Comput. 11 (1) (1998) 91-101.

[2] W. Gerstner, Population dynamics for spiking neurons: fast transients, asynchronous states and locking, Neural Comput 12 (2000) 43-89.

[3] W. Gerstner, J.L. Van Hemmen, J.D. Cowan, What matters in neuronal locking, Neural Comput. 8 (1996) 1689-1712.

[4] B.W. Knight, Dynamics of encoding in a population of neurons, J. Gen. Physiol. 59 (1972) 734-766.

[5] D.J. Mar, C.C. Chow, W. Gerstner, R.W. Adams, J.J. Collins, Noise-shaping in populations of coupled model neurons, Proc. Natl. Acad. Sci. USA 96 (1999) 10450-10 455.

[6] H.E. Plesser, W. Gerstner, Noise in integrate-and-fire neurons: from stochastic input to escape rates, Neural Comput 12 (2000) 367-384.

[7] W.R. Softky, C. Koch, The highly irregular firing of cortical cells is inconsistent with temporal intergration of random EPSPs, J. Neurosci. 13 (1993) 334-350.

[8] M. Spiridon, W. Gerstner, Noise spectrum and signal transmission through a population of spiking neurons, Network 10 (1999) 257-272. 
[9] S. Thorpe, D. Fize, C. Marlot, Speed of processing in the human visual system, J. Comput. Neurosci. 381 (1996) 520-522.

[10] A. Treves, Mean-field analysis of neuronal spike dynamics, Network 4 (1993) 259-284.

[11] M.V. Tsodyks, T. Sejnowski, Rapid state switching in balanced cortical network models, Comput. Neural Systems 6 (1995) 111-124.

[12] H. Yoon, H. Sompolinsky, Population coding with correlated noise, in: Michael I. Jordan, Michael J. Kearns, Sara A. Solla (Eds.), Advances in Neural Information Processing Systems, Vol. 11, MIT Press, Cambridge, MA, 1999.

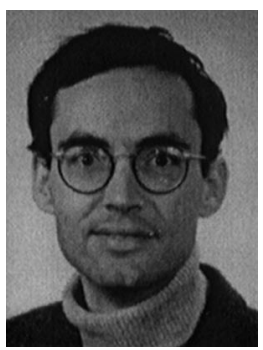

Wulfram Gerstner is Assistant Professor and Director of the Center for Neuromimetic Systems MANTRA at the EPFL. His research interests are in the theory of neural network with a special emphasis on models of spiking neurons, on the problem of temporal coding, and on the role of spatial representation for navigation and path planning of autonomous agents. He teaches courses on Neural Networks for Computer Scientists, Electrical Engineers, and Physicists at the EPFL.

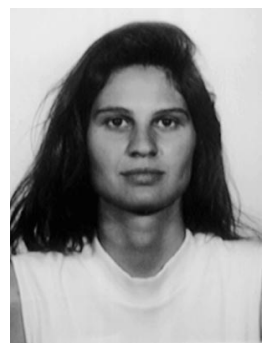

Mona Spiridon studied physics at the Swiss Federal Institute of Technology Lausanne (EPFL), Switzerland. She graduated in March 1995. She received a M.Sc. in Physiology from University College London in 1996. She is currently working towards a Ph.D. at the Center for Neuromimetic Systems, EPFL Lausanne, Switzerland. Her current research interests are population coding methods and the reliability of signal transmission in a neuronal population.

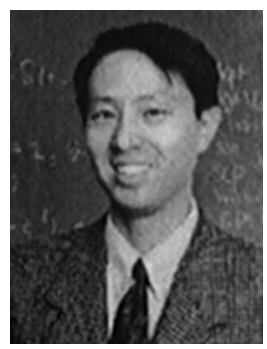

Carson Chow obtained his Ph.D. in 1992 from the Massachusetts Institute of Technology. He then went on to post-doctoral appointments at the University of Colorado and Boston University before taking his present position as Assistant Professor of Mathematics at the University of Pittsburgh. 